Cell Survival in Corneal Endothelial Dystrophies Rajalekshmy Shyam ${ }^{1}$, Diego Ogando ${ }^{2}$, Moonjung Choi $^{2}$ and Joseph Bonanno ${ }^{2}$

${ }^{1}$ Indiana University School of Medicine and ${ }^{2}$ Indiana University Bloomington

OBJECTIVES/SPECIFIC AIMS: Purpose - The goal of this study is to understand how loss of the membrane protein SLC4A11 alters endothelial cell metabolism thereby producing Corneal Endothelial Dystrophy. Studies from our lab indicated that glutamine-dependent mitochondrial dysfunction is one of the outcomes of SLC4a11 loss. In the current study, we ask if autophagy and mitophagy pathways and the signaling pathways that regulate these processes are altered in SLC4a11 KO cells. METHODS/STUDY POPULATION: Methods Immortalized mouse WT and SLC4a11 KO cell lines were incubated in DMEM with and without $0.5 \mathrm{mM}$ glutamine for 6 hours. In order to assess mitophagy, cells were stained using Lysotracker Red and Mitotracker Green. Colocalization co-efficients of red and green channels were obtained for at least 35 cells using ZeissZen Pro software. Student's t-test was used to determine statistical significance. For Western Blots, antibodies against LC3b, AMPK, pAMPK, and b-actin were used to examine autophagy flux and potential signaling pathways that regulate autophagy. RESULTS/ ANTICIPATED RESULTS: Results - In the presence of glutamine, the colocalization co-efficient of Lysotracker Red and Mitotracker Green channels was significantly increased in KO cells $(0.74 \pm 0.18)$ relative to WT $(0.58 \pm 0.20)$ with a $\mathrm{p}$-value $\leq 0.0024$. In the absence of glutamine, the colocalization co-efficient was reversed, for $\mathrm{KO}$ cells $0.54 \pm 0.14$ and for WT cells $0.77 \pm 0.0 .16$ with a p-value $\leq 0.0001$, suggesting increased mitophagy by glutamine in KO cells. Western Blots indicated that glutamine increased autophagy flux, as indicated by increased levels of LC3b following bafilomycin A treatment in $\mathrm{KO}$ cells. Concomitantly, there was an increase in pAMPK/AMPK levels suggesting a potential mechanism for increased mitophagy. DISCUSSION/SIGNIFICANCE OF IMPACT: Conclusion and Future studies -Our data indicates enhanced mitophagy as well as autophagy in SLC4a11 KO cells. Future studies will determine whether these processes regulate cell survival in mouse models of corneal endothelial dystrophies.

\section{Characterizing the Neural Signature of Metabolic Syndrome}

Eithan Kotkowski ${ }^{1}$, Larry R. Price ${ }^{1}$, Crystal G. Franklin ${ }^{1}$, Maximino Salazar ${ }^{1}$, Ralph A. DeFronzo ${ }^{2}$, David Glahn ${ }^{3}$, John Blangero ${ }^{4}$ and Peter T. Fox ${ }^{1}$

${ }^{1}$ University of Texas Health Science Center San Antonio: Research Imaging Institute; ${ }^{2}$ UTHSA: Diabetes Division, Texas Diabetes

Institute; ${ }^{3}$ Yale School of Medicine and ${ }^{4}$ UTRGV School of Medicine

OBJECTIVES/SPECIFIC AIMS: Our objective is to understand the influence of the features comprising metabolic syndrome (central obesity, raised fasting plasma glucose, triglycerides, blood pressure, and decreased HDL cholesterol) on brain structure in men and women. With the understanding that MetS is a strong predictor of gray matter volume loss in specific brain regions, in this study we sought to quantify the influence of each of the metabolic syndrome biometric variables on the structures involved in the neural signature of metabolic syndrome. METHODS/STUDY POPULATION: We conducted multiple linear regression analyses on a cross-sectional sample of 800 individuals from the Genetics of Brian Structure (GOBS) image archive (352 men and 448 women). GOBS is an offshoot of the San Antonio Heart Study involving an extended pedigree of Mexican Americans from the greater San Antonio area. Its goal is to localize, identify, and characterize genes/quantitative trait loci associated with variations in brain structure and function (Winkler, 2010). The archive has continuously added participants from approximately 40 families since 2006. Neuroanatomic (T1-weighted MRI scans obtained on a Siemens 3T scanner and processed using FSL), neurocognitive, and biometric phenotypes have been obtained for each subject (including blood lipids). Linear regressions were run using SPSS and incorporated biometric and gray matter volume values obtained from 800 GOBS participants. RESULTS/ANTICIPATED RESULTS: Linear regressions incorporating metabolic syndrome variables as dependent variables and gray matter volume from regions involved in the neural signature of metabolic syndrome as predictors show significant predictive patterns that are largely similar between men and women, with some differences. Another linear regression conducted with gray matter volume from the neural signature of metabolic syndrome as the dependent variable and metabolic syndrome variables as predictors show that waist circumference and triglycerides are the greatest predictors of gray matter volume loss in men, and fasting plasma glucose and waist circumference are the greatest predictors of gray matter volume loss in women. DISCUSSION/SIGNIFICANCE OF IMPACT: Significant sex differences in the relationships between metabolic syndrome variables and gray matter volume changes between brain regions comprising the neural signature of metabolic syndrome were identified. waist circumference, fasting plasma glucose, and triglycerides are the most reliable predictors of gray matter volume loss. The variance in gray matter volume of the neural signature of metabolic syndrome in men is more significantly explained by waist circumference and triglycerides (when accounting for age) and in women is more significantly explained by waist circumference and fasting plasma glucose (when accounting for age). A model of metabolic syndrome that emphasizes a risk of neurodegeneration should focus on waist circumference for both men and women and weigh the remaining variables accordingly by sex (triglycerides in men and fasting plasma glucose in women).

3375

\section{Chronic inflammation promotes intestinal macrophages} to become modulators of the Notch pathway

Eliseo Castillo

University of New Mexico Clinical and Translational Science Center

OBJECTIVES/SPECIFIC AIMS: The purpose of this research was to investigate how chronic inflammation promotes the generation of proinflammatory intestinal macrophages and if macrophages contribute to intestinal inflammation through Notch activation. METHODS/STUDY POPULATION: We utilized two animal models of chronic colitis, the chronic DSS-induced colitis mouse model and the spontaneous enterocolitis development in IL-10-deficient mice to investigate the role of chronic inflammation in the generation of proinflammatory intestinal macrophages and its influence in notch signaling. Bone marrow-derived monocytes were collected from each group and differentiated into macrophages (BMM) for gene and protein analysis. Ex vivo phenotypical and functional 